\title{
Cloaking via Change of Variables for the Helmholtz Equation in the Whole Space
}

\author{
HOAI-MINH NGUYEN \\ Courant Institute
}

\begin{abstract}
This paper is devoted to the study of a cloaking device that is composed of a standard near cloak based on a regularization of the transformation optics, i.e., a change of variables that blows up a small ball to the cloaked region, and a fixed lossy layer for the Helmholtz equation in the whole space of dimension 2 or 3 with the outgoing condition at infinity. We establish a degree of near invisibility, which is independent of the content inside the cloaked region, for this device. We also show that the lossy layer is necessary to ensure the validity of the degree of near invisibility when no constraint on physical properties inside the cloaked region is imposed. (c) 2010 Wiley Periodicals, Inc.
\end{abstract}

\section{Introduction}

This paper concerns an approach to cloaking introduced by Greenleaf, Lassas, and Uhlmann [5], Pendry, Schurig, and Smith [15], and Leonhardt [11]. Their "transformation optics" scheme uses a singular change of coordinates that blows up a point to a cloaked region. Although this approach is excellent in many aspects, it has the defect that one needs to work with the singular structure. This implies difficulties in practice as well as theory; see, e.g., [3, 19].

Recently, to avoid working with this singular structure, Ruan et al. [16] and Kohn et al. [8] suggested studying a regularization of this approach by considering a near cloaking based on a change of coordinates that blows up a small ball to the cloaked region. In [16] Ruan et al. studied this problem for scattered waves of the Helmholtz equation in two dimensions where the cloaked region is a ball and the material inside the cloaked region is homogeneous. They argued that in the limiting case, the standard near cloak should become a perfect one. In [8] Kohn et al. studied a near invisibility for the Neumann-to-Dirichlet map in the context of electric impedance tomography using a standard near cloak. They showed that the near cloaking is achieved for an arbitrary material inside the cloaked region.

In [12] Liu studied this issue for the Helmholtz equation in the whole space of dimension 2 or 3 with the outgoing condition at infinity. Using a standard near cloak, imposing the Dirichlet boundary condition at the boundary of the cloaked region, and considering scattered waves generated by plane waves, he established a near

Communications on Pure and Applied Mathematics, Vol. LXIII, 1505-1524 (2010)

(C) 2010 Wiley Periodicals, Inc. 
invisibility for the far-field pattern. In [7] Kohn et al. investigated a near cloaking of the Dirichlet-to-Neumann map for the Helmholtz equation in a bounded domain of dimension 2 or 3 . Because of the bounded domain, they have to assume some condition on the frequency so that their problem is well-posed. Their near cloak contains a lossy layer between a standard one and the cloaked region. They proved that their near cloaking is achieved regardless of the content inside the cloaked region.

This paper is devoted to the study of a near cloaking for the Helmholtz equation in the whole space of dimension 2 or 3 for an arbitrary geometry object, with the transmission conditions at interfaces of different materials and with a general source outside the cloaked region. Our near cloak contains a standard one based on the change-of-variable scheme mentioned above and a (fixed) lossy layer between the standard one and the cloaked region that is independent of the parameter of regularization $\varepsilon$. Here the parameter of regularization $\varepsilon$ is defined as the rate between the diameter of a region that blows up to the cloaked region and the diameter of the cloaked region. We show that the degree of near invisibility for the near field is $\varepsilon$ in three dimensions and $1 /|\log \varepsilon|$ in two (Theorem 1.2). This is compatible with the results in $[7,12]$.

Our estimate is uniform with respect to the material inside the cloaked region (see Theorem 1.2). Moreover, we also prove that to achieve such uniformity, the existence of the lossy layer is necessary (see Theorem 1.7) although our problem is well-posed despite the existence of a lossy layer. Our method of proof of degree of near invisibility is completely different from the ones used in the works mentioned above. It is based on an idea in [14] and standard techniques presented in [10, 21]. To prove the necessity of the lossy layer, we make use of special functions in the Helmholtz setting. Our work is strongly motivated by that of Kohn et al. [7].

Let us describe our problem mathematically. To illustrate the idea, instead of defining cloaks for arbitrary geometry objects, let us explain what it means for a specific structure $a_{c}, \sigma_{c}$ defined in the annular $\frac{1}{2}<|x|<2$ to cloak the ball $B_{1 / 2}$ of $\mathbb{R}^{d}(d=2,3)$. Hereafter $B$ denotes the unit open ball of $\mathbb{R}^{d}$ and $D_{r}$ denotes the set $\{r x: x \in D\}$ for any convex set $D$ containing the origin and for any $r>0$. Let $u_{c} \in H_{\mathrm{loc}}^{1}\left(\mathbb{R}^{d}\right)$ be the unique solution of the Helmholtz equation

$$
\operatorname{div}\left(A_{c}(x) \nabla u_{c}\right)+k^{2} \Sigma_{c}(x) u_{c}=f \quad \text { in } \mathbb{R}^{d},
$$

where

$$
A_{c}, \Sigma_{c}= \begin{cases}I, 1 & \text { in } \mathbb{R}^{d} \backslash B_{2}, \\ a_{c}(x), \sigma_{c}(x) & \text { in } B_{2} \backslash B_{1 / 2}, \\ a(x), \sigma(x) & \text { in } B_{1 / 2},\end{cases}
$$

such that $u_{c}$ satisfies the well-known outgoing condition, i.e.,

$$
\begin{cases}\frac{\partial u_{c}}{\partial r}-i k u_{c}=O\left(1 / r^{\frac{3}{2}}\right) & \text { as } r=|x| \rightarrow \infty \text { if } d=2, \\ \frac{\partial u_{c}}{\partial r}-i k u_{c}=O\left(1 / r^{2}\right) & \text { as } r=|x| \rightarrow \infty \text { if } d=3 .\end{cases}
$$


Here $a_{c}$ and $a$ are matrix functions that are uniformly elliptic, $\sigma_{c}$ and $\sigma$ are complex

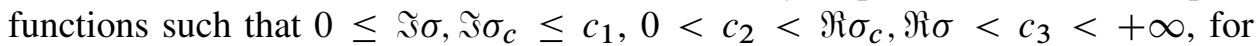
some $c_{1}, c_{2}$, and $c_{3}$ on their domain of definition, and $f \in L^{2}\left(\mathbb{R}^{d}\right)$ such that supp $f \subset\left(B_{4} \backslash B_{3}\right)$. Physically $a_{c}$ and $\sigma_{c}$ describe the material properties in the cloaking device $B_{2} \backslash B_{1 / 2} ; a$ and $\sigma$ describe the material properties in the cloaked region $B_{1 / 2}$; and $f$ is a given source outside the cloaking device and the cloaked region. Let $u \in H_{\text {loc }}^{1}\left(\mathbb{R}^{d}\right)$ be the unique solution of

$$
\Delta u+k^{2} u=f \quad \text { in } \mathbb{R}^{d}
$$

such that $u$ satisfies the outgoing condition.

To cloak perfectly, we need to construct $a_{c}$ and $\sigma_{c}$ such that $u_{c}=u$ outside $B_{2}$. To have a near cloaking, we need to construct $a_{c}$ and $\sigma_{c}$ such that $u_{c} \approx u$ outside $B_{2}$. Before giving the formulas for $a_{c}$ and $\sigma_{c}$ in these situations, let us recall the following basic fact on which the change-of-variable-based cloaking scheme relies.

PROPOSITION 1.1 Let $d \geq 2$, A be a matrix function, and $\Sigma$ be a complex function defined on $\mathbb{R}^{d}, F: \mathbb{R}^{d} \rightarrow \mathbb{R}^{d}$ be Lipschitz, surjective, and invertible, and $f \in$ $L^{2}\left(\mathbb{R}^{d}\right)$ such that $\|A\|_{L^{\infty}}<+\infty,\|\Sigma\|_{L^{\infty}}<+\infty, F(x)=x$ on $\mathbb{R}^{d} \backslash B_{2}, F^{-1}$ is Lipschitz, det $D F>c$ a.e., $x \in \mathbb{R}^{d}$, det $D F^{-1}>c$ a.e., $x \in \mathbb{R}^{d}$ for some positive constant $c>0$, and $\operatorname{supp} f \subset B_{4} \backslash B_{3}$. Then $u \in H_{\mathrm{loc}}^{1}\left(\mathbb{R}^{d}\right)$ is a solution of

$$
\operatorname{div}(A \nabla u)+k^{2} \Sigma u=f \quad \text { in } \mathbb{R}^{d}
$$

if and only if $v:=u \circ F^{-1} \in H_{\mathrm{loc}}^{1}\left(\mathbb{R}^{d}\right)$ is a solution of

$$
\operatorname{div}\left(F_{*} A \nabla v\right)+k^{2} F_{*} \Sigma v=f \quad \text { in } \mathbb{R}^{d} .
$$

Here

$$
F_{*} A(y)=\frac{D F(x) A(x) D F^{\top}(x)}{\operatorname{det} D F(x)}, \quad F_{*} \Sigma(y)=\frac{\Sigma(x)}{\operatorname{det} D F(x)}, \quad x=F^{-1}(y) .
$$

Moreover, $u=v$ outside $B_{2}$.

We are ready to give the construction of $a_{c}$ and $\sigma_{c}$. For the perfect cloaking case, a standard construction is

$$
A_{c}, \Sigma_{c}= \begin{cases}I, 1 & \text { in } \mathbb{R}^{d} \backslash B_{2}, \\ G_{*} I, G_{*} 1 & \text { in } B_{2} \backslash B_{1 / 2}, \\ a, \sigma & \text { in } B_{1 / 2},\end{cases}
$$

where $G=\lim _{\varepsilon \rightarrow 0} G_{\varepsilon}$ and $G_{\varepsilon}$ is defined by

$$
G_{\varepsilon}= \begin{cases}x & \text { if } x \in \mathbb{R}^{d} \backslash B_{2}, \\ \left(\frac{1-2 \varepsilon}{2-\varepsilon}+\frac{3|x|}{2(2-\varepsilon)}\right) \frac{x}{|x|} & \text { if } x \in B_{2} \backslash B_{\varepsilon}, \\ \frac{x}{2 \varepsilon} & \text { if } x \in B_{\varepsilon} .\end{cases}
$$

We note that $G_{\varepsilon}$ maps $\partial B_{2}$ and $\partial B_{\varepsilon}$ into $\partial B_{2}$ and $\partial B_{1 / 2}$, respectively. It is clear to see that the construction is singular at 0 . 
To obtain a near cloaking, we use the following construction:

$$
A_{c}, \Sigma_{c}= \begin{cases}I, 1 & \text { in } \mathbb{R}^{d} \backslash B_{2}, \\ F_{\varepsilon *} I, F_{\varepsilon *} 1 & \text { in } B_{2} \backslash B_{1}, \\ I, 1+\frac{i}{k} & \text { in } B_{1} \backslash B_{1 / 2}, \\ a, \sigma & \text { in } B_{1 / 2},\end{cases}
$$

where

$$
F_{\varepsilon}= \begin{cases}x & \text { if } x \in \mathbb{R}^{d} \backslash B_{2}, \\ \left(\frac{2-2 \varepsilon}{2-\varepsilon}+\frac{|x|}{2-\varepsilon}\right) \frac{x}{|x|} & \text { if } x \in B_{2} \backslash B_{\varepsilon}, \\ \frac{x}{\varepsilon} & \text { if } x \in B_{\varepsilon} .\end{cases}
$$

We note that $F_{\varepsilon}$ maps $\partial B_{2}$ and $\partial B_{\varepsilon}$ into $\partial B_{2}$ and $\partial B_{1}$, respectively.

The effectiveness of this near cloak is confirmed in Section 2.2. The main result proved there is the following:

THEOREM 1.2 Let $d=2,3, k>0,0<\varepsilon<\frac{1}{4}, f \in L^{2}\left(\mathbb{R}^{d}\right)$, a be a measurable matrix function, and $\sigma$ be a measurable complex function defined on the ball $B_{1 / 2}$ of $\mathbb{R}^{d}$. Assume that supp $f \subset B_{4} \backslash B_{3}$, a is uniformly elliptic, and $0 \leq \Im(\sigma) \leq c_{1}$ and $0<c_{2}<\Re(\sigma)<c_{3}<+\infty$ for some constants $c_{1}, c_{2}$, and $c_{3}$ on $B_{1 / 2}$. Let $u_{c}$ and $u$ in $H_{\mathrm{loc}}^{1}\left(\mathbb{R}^{d}\right)$ be the solutions of (1.1) and (1.2), respectively, where $\left(A_{c}, \Sigma_{c}\right)$ is defined by (1.3) such that $u_{c}$ and $u$ satisfy the outgoing condition. Then

$$
\begin{cases}\left\|u_{c}-u\right\|_{H^{1}\left(B_{3} \backslash B_{2}\right)} \leq \frac{C}{|\ln \varepsilon|}\|f\|_{L^{2}} & \text { if } d=2, \\ \left\|u_{c}-u\right\|_{H^{1}\left(B_{3} \backslash B_{2}\right)} \leq C \varepsilon\|f\|_{L^{2}} & \text { if } d=3,\end{cases}
$$

for some positive constant $C$ depending on $k$ but independent of $\varepsilon, f, a, \sigma, c_{1}, c_{2}$, and $c_{3}$.

Remark 1.3. Our construction could be used to hide an arbitrary physical object in the cloaked region, since our estimate is independent of $a$ and $\sigma$. In other words, our near cloaking is achieved regardless of the content of the cloaked region.

Remark 1.4. Theorem 1.2 is a consequence of Proposition 1.1 and Theorem 2.1 where we deal with an arbitrary geometry object. Hence our method can be applied to cloak a region of arbitrary shape.

Remark 1.5. In [7], Kohn et al. studied a similar problem for the Helmholtz equation in a bounded domain. All of us use a lossy layer in our construction. Our method of proof is different from theirs and is also simpler, particularly for the three-dimensional case.

Remark 1.6. Estimate (1.5) also holds for some constant $C$ independent of $\alpha$ (see the proof of Theorem 1.2) if we take

$$
A_{c}, \Sigma_{c}=I, 1+\frac{i \alpha}{k}
$$


(for any $\alpha>1$ ) in $B_{1} \backslash B_{1 / 2}$. This yields the result for the Dirichlet condition at the inner edge of a standard cloaking device. Thus we rediscover and improve the result of Liu [12] since we only use here a fixed material, and moreover our estimate is established for the near field.

In Section 2.3 we show that it is necessary to have a lossy layer besides a standard near cloak based on the change-of-variable scheme if we want to obtain a near cloaking regardless of the material inside the cloaked region. The main result of Section 2.3 is the following:

THEOREM 1.7 Let $d=2,3$. Then for any $0<\varepsilon<\frac{1}{4}, r>4$, and $\lambda>0$, there exist two positive constants $a$ and $\sigma$ such that

$$
\left\|u_{s}\right\|_{L^{2}\left(B_{r} \backslash B_{2}\right)} \geq \lambda
$$

Here $u_{s} \in H_{\mathrm{loc}}^{1}\left(\mathbb{R}^{d}\right)$ is such that $u_{s}$ satisfies the outgoing condition and $u:=$ $u_{s}+e^{i k x_{d}}$ is a solution of the Helmholtz equation

$$
\operatorname{div}\left(A_{c} \nabla u\right)+k^{2} \Sigma_{c} u=0
$$

where $\left(A_{c}, \Sigma_{c}\right)$ is defined as follows:

$$
A_{c}, \Sigma_{c}= \begin{cases}I, 1 & \text { in } \mathbb{R}^{d} \backslash B_{2}, \\ G_{\varepsilon *} I, G_{\varepsilon *} 1 & \text { in } B_{2} \backslash B_{1 / 2}, \\ a, \sigma & \text { in } B_{1 / 2} .\end{cases}
$$

Remark 1.8. Kohn et al. considered a near cloaking via a change of variables of the Dirichlet-to-Neumann map for the Helmholtz equation in a bounded domain. They showed that in their setting a lossy layer is necessary to obtain an estimate of degree of near invisibility regardless of the content inside the cloaked region. In fact, they showed that for any small $\varepsilon$, the parameter of regularization, and for any frequency $k$, there exists a material inside the cloaked region such that the associated problem is not well-posed. Our situation is different from theirs. Our problem is well-posed for any $\varepsilon$ and $k$ and for any material inside the cloaked region. Nevertheless, the uniformity of the degree of near invisibility does not hold without a lossy layer.

Finally, we want to mention the works of Greenleaf et al. in [3] (see also the work of Hetmaniuk, Liu, and Uhlmann in [6]) and Weder in [18]. They worked directly with the (singular) scheme and introduced weak notions of solutions for this scheme. It would be nice to verify that these notions are consistent. The reader interested in cloaking can find a recent survey on this subject in [4]. 


\section{Proof of the Main Results}

\subsection{Change of Variables for Helmholtz Equations; Proof of Proposition 1.1}

The proof of Proposition 1.1 is elementary. It is based on the variational formula of the solutions. Indeed, $u$ is characterized by the fact that $u \in H_{\mathrm{loc}}^{1}\left(\mathbb{R}^{d}\right)$ and

$$
\int_{\mathbb{R}^{d}}\left(A \nabla u \nabla \phi-k^{2} \Sigma u \phi\right) d x=-\int_{\mathbb{R}^{d}} f \phi d x
$$

for all Lipschitz $\phi$ with compact support. Replacing $x$ by $F^{-1}(y)$, we have

$$
\int_{\mathbb{R}^{d}}\left(F_{*} A \nabla v \nabla \varphi-k^{2} F_{*} \Sigma v \varphi\right) d y=-\int_{\mathbb{R}^{d}} f \varphi d y,
$$

with $\varphi=\phi \circ F^{-1}$, since supp $f \subset B_{4} \backslash B_{3}$ and $F(x)=x$ for $x \in \mathbb{R}^{d} \backslash B_{2}$. This implies, since $\phi$ is arbitrary,

$$
\int_{\mathbb{R}^{d}}\left(F_{*} A \nabla v \nabla \varphi-k^{2} F_{*} \Sigma v \varphi\right) d y=-\int_{\mathbb{R}^{d}} f \varphi d y
$$

for all Lipschitz $\varphi$ with compact support. This implies all conditions on $v$.

To prove the inverse, it suffices to note that

$$
F_{*}^{-1}\left(F_{*} A\right)=A \text { and } F_{*}^{-1}\left(F_{*} \Sigma\right)=\Sigma .
$$

\subsection{Study of a Small Inclusion; Proof of Theorem 1.2}

It is important to note that if $\left(A_{c}, \Sigma_{c}\right)$ is defined by (1.3), then $A_{c}=F_{\varepsilon *} A_{\varepsilon}$ and $\Sigma_{c}=F_{\varepsilon *} \Sigma_{\varepsilon}$ where

$$
A_{\varepsilon}, \Sigma_{\varepsilon}= \begin{cases}I, 1 & \text { if } x \in \mathbb{R}^{d} \backslash B_{\varepsilon}, \\ \frac{1}{\varepsilon^{d-2}} I, \frac{1}{\varepsilon^{d}}\left[1+\frac{i}{k}\right] & \text { if } x \in B_{\varepsilon} \backslash B_{\varepsilon / 2}, \\ \frac{1}{\varepsilon^{d-2}} a\left(\frac{x}{\varepsilon}\right), \frac{1}{\varepsilon^{d}} \sigma\left(\frac{x}{\varepsilon}\right) & \text { if } x \in B_{\varepsilon / 2} .\end{cases}
$$

Using this observation and applying Proposition 1.1, Theorem 1.2 is a consequence of the following:

TheOREM 2.1 Let $d=2,3, k>0,0<\varepsilon<\frac{1}{4}, D \subset B_{1}$ be a smooth, open, convex subset of $\mathbb{R}^{d}, f \in L^{2}\left(\mathbb{R}^{d}\right)$, a be a measurable matrix function, and $\sigma$ be a measurable complex function defined on $D_{1 / 2}$. Assume that supp $f \subset B_{4} \backslash B_{3}$, a is uniformly elliptic, and $0 \leq \Im(\sigma) \leq c_{1}<\infty$ and $0<c_{2}<\Re(\sigma)<c_{3}<+\infty$ for some $c_{1}, c_{2}$, and $c_{3}$ on $D_{1 / 2}$. Define

$$
A_{\varepsilon}, \Sigma_{\varepsilon}= \begin{cases}I, 1 & \text { if } x \in \mathbb{R}^{d} \backslash D_{\varepsilon}, \\ \frac{1}{\varepsilon^{d-2}} I, \frac{1}{\varepsilon^{d}}\left[1+\frac{i}{k}\right] & \text { if } x \in D_{\varepsilon} \backslash D_{\varepsilon / 2}, \\ \frac{1}{\varepsilon^{d-2}} a\left(\frac{x}{\varepsilon}\right), \frac{1}{\varepsilon^{d}} \sigma\left(\frac{x}{\varepsilon}\right) & \text { if } x \in D_{\varepsilon / 2} .\end{cases}
$$


Let $u_{\varepsilon} \in H_{\mathrm{loc}}^{1}\left(\mathbb{R}^{d}\right)$ be the unique solution of

$$
\left\{\begin{array}{l}
\nabla \cdot\left(A_{\varepsilon} \nabla u_{\varepsilon}\right)+k^{2} \Sigma_{\varepsilon} u_{\varepsilon}=f \quad \text { in } \mathbb{R}^{d}, \\
u_{\varepsilon} \text { satisfies the outgoing condition. }
\end{array}\right.
$$

Then there exists a constant $C=C(k, D)$, independent of $\varepsilon, f, a, \sigma, c_{1}, c_{2}$, and $c_{3}$, such that

$$
\begin{cases}\left\|u_{\varepsilon}-u\right\|_{H^{1}\left(B_{3} \backslash B_{2}\right)} \leq \frac{C}{|\ln \varepsilon|}\|f\|_{L^{2}} & \text { if } d=2, \\ \left\|u_{\varepsilon}-u\right\|_{H^{1}\left(B_{3} \backslash B_{2}\right)} \leq C \varepsilon\|f\|_{L^{2}} & \text { if } d=3 .\end{cases}
$$

Here $u$ is the unique solution of (1.2).

In this section, we deal with some estimates for Helmholtz equations. Our method of proof is based on and inspired from the standard variational one presented in $[10,21]$. The integral equation method (see, e.g., [1, 13]) may not be appropriate for our setting where its parameters $\left(A_{\varepsilon}, \sigma_{\varepsilon}\right)$ are not piecewise constant.

The following lemma concerns the phenomena of low frequency, which naturally appears in the study of Theorem 2.1.

LEMMA 2.2 Let $d=2,3, k>0,0<\varepsilon<k, D \subset B_{1}$ be a smooth open subset of $\mathbb{R}^{d}$, and $g \in H^{1 / 2}(\partial D)$. Assume that $\mathbb{R}^{d} \backslash D$ is connected and $v_{\varepsilon} \in H_{\mathrm{loc}}^{1}\left(\mathbb{R}^{d}\right)$ is the unique solution of

$$
\begin{cases}\Delta v_{\varepsilon}+\varepsilon^{2} v_{\varepsilon}=0 & \text { in } \mathbb{R}^{d} \backslash D, \\ v_{\varepsilon}=g & \text { on } \partial D, \\ v_{\varepsilon} \text { satisfies the outgoing condition. } & \end{cases}
$$

Then

$$
\left\|v_{\varepsilon}\right\|_{H^{1}\left(B_{r} \backslash D\right)} \leq C_{r}\|g\|_{H^{1 / 2}(\partial D)} \quad \forall r>5
$$

and

$$
\begin{cases}\left\|v_{\varepsilon}\right\|_{L^{2}\left(B_{4 / \varepsilon} \backslash B_{1 / \varepsilon}\right)} \leq \frac{C}{\varepsilon^{1 / 2}|\log \varepsilon|}\|g\|_{H^{1 / 2}(\partial D)} & \text { if } d=2, \\ \left\|v_{\varepsilon}\right\|_{L^{2}\left(B_{4 / \varepsilon} \backslash B_{1 / \varepsilon}\right)} \leq C\|g\|_{H^{1 / 2}(\partial D)} & \text { if } d=3,\end{cases}
$$

for some positive constants $C_{r}=C(r, k, D)$ and $C=C(k, D)$.

Remark 2.3. Lemma 2.2 is standard in the case $d=3$ (see, e.g., [10]). In the case $d=2$, some difficulties in the investigation of the stability of the solutions appear as mentioned in [2, p. 66] since the fundamental solution of the Helmholtz equation, which satisfies the outgoing condition, does not converge as the frequency goes to 0. In this case, (2.3) was proved by Werner in [20] and Kress in [9] by the integral equation method; (2.4) should be known but we cannot find any reference for it. In this paper, we use an approach, based on the method presented in $[10,21]$ 
to obtain the whole conclusion of Lemma 2.2. In the case $d=2$, our key ingredients are the following properties of $H_{l}$, the Hankel function of the first kind of order $l$; see, e.g., [2, (3.61), p. 66] and [17, p. 446],

$$
\lim _{r \rightarrow 0} \frac{1}{|\ln r|} H_{0}(r)=\frac{2}{i \pi}, \quad\left|H_{0}\right| \searrow \text { on } \mathbb{R}_{+}, \quad \lim _{r \rightarrow 0} \frac{r d H_{0}(r)}{d r}=-\frac{2}{i \pi}
$$

and

$$
\int_{\partial B_{t}}\left|H_{l}\right|^{2} \leq \int_{\partial B_{s}}\left|H_{l}\right|^{2} \quad \forall 0<s<t, \forall l \neq 0 .
$$

ProOF: Here we give the proof only in the case $d=2$. We first prove that

$$
\left\|v_{\varepsilon}\right\|_{L^{2}\left(B_{5} \backslash D\right)} \leq C\|g\|_{H^{1 / 2}(\partial D)}
$$

for some positive constant $C$ depending only on $k$ and $D$ by contradiction. Suppose that this is not true. Then there exist a sequence $\varepsilon_{n} \rightarrow 0_{+}$and a sequence $g_{n} \in H^{1 / 2}(\partial D)$ such that

$$
\lim _{n \rightarrow \infty}\left\|g_{n}\right\|_{H^{1 / 2}(\partial D)}=0 \quad \text { and } \quad\left\|v_{n}\right\|_{L^{2}\left(B_{5} \backslash D\right)}=1,
$$

where $v_{n} \in H_{\text {loc }}^{1}\left(\mathbb{R}^{2} \backslash D\right)$ is the unique solution of

$$
\begin{cases}\Delta v_{n}+\varepsilon_{n}^{2} v_{n}=0 & \text { in } \mathbb{R}^{2} \backslash \bar{D}, \\ v_{n}=g_{n} & \text { on } \partial D, \\ v_{n} \text { satisfies the outgoing condition. } & \end{cases}
$$

Since $\left\|v_{n}\right\|_{L^{2}\left(B_{5} \backslash D\right)}=1, \Delta v_{n}+\varepsilon_{n}^{2} v_{n}=0$ in $\mathbb{R}^{2} \backslash B_{1}$, and $v_{n}$ satisfies the outgoing condition, it follows that $v_{n}$ can be represented by

$$
v_{n}(x)=\sum_{l=-\infty}^{\infty} a_{l, n} H_{l}\left(\varepsilon_{n}|x|\right) e^{i l \theta} \quad \forall x:|x|>1 .
$$

Then

$$
v_{n}=v_{0, n}+v_{1, n}
$$

where

$$
v_{0, n}=a_{0, n} H_{0}\left(\varepsilon_{n}|x|\right) \quad \text { and } \quad v_{1, n}=\sum_{l \neq 0} a_{l, n} H_{l}\left(\varepsilon_{n}|x|\right) e^{i l \theta} .
$$

Since $\left(e^{i l \theta}\right)_{l=-\infty}^{\infty}$ is orthogonal in $L^{2}\left(\partial B_{1}\right)$ and $\left\|v_{n}\right\|_{L^{2}\left(B_{5} \backslash D\right)}=1$, it follows from (2.5), (2.6), and (2.11) that

$$
\left|a_{0, n}\right| \leq \frac{C}{\left|\ln \varepsilon_{n}\right|}
$$

and

$$
\int_{\partial B_{r}}\left|v_{1, n}\right|^{2} \leq C \quad \forall r>2
$$


From (2.9), we have

$$
\int_{B_{5} \backslash D}\left|\nabla v_{n}\right|^{2}-\varepsilon_{n}^{2} \int_{B_{5} \backslash D}\left|v_{n}\right|^{2}=\int_{\partial B_{5}} \frac{\partial v_{n}}{\partial r} \bar{v}_{n}-\int_{\partial D} \frac{\partial v_{n}}{\partial \eta} \bar{g}_{n}
$$

Thus since $\nabla v_{n}+\varepsilon_{n}^{2}=0$ in $\mathbb{R}^{2} \backslash D$ and $\lim _{n \rightarrow \infty}\left\|g_{n}\right\|_{H^{1 / 2}(\partial D)}=0$, it follows from (2.5), (2.6), (2.12), and (2.13) that

$$
\int_{B \backslash \backslash D}\left|\nabla v_{n}\right|^{2} \leq C
$$

Hence since $\Delta v_{n}+\varepsilon_{n}^{2} v_{n}=0$, without loss of generality, we may assume that $v_{n} \rightarrow v$ weakly in $H_{\mathrm{loc}}^{1}\left(\mathbb{R}^{2} \backslash D\right)$ and $v_{n} \rightarrow v$ in $L^{2}\left(B_{5} \backslash D\right)$.

We next prove that $\int_{\mathbb{R}^{2} \backslash D}|\nabla v|^{2}<+\infty$. We have

$$
\int_{B_{m} \backslash D}|\nabla v|^{2} \leq \liminf _{n \rightarrow \infty} \int_{B_{m} \backslash D}\left|\nabla v_{n}\right|^{2} \quad \forall m>2 .
$$

On the other hand, by (2.9),

$$
\int_{B_{m} \backslash D}\left|\nabla v_{n}\right|^{2} \leq \varepsilon_{n}^{2} \int_{B_{m} \backslash D}\left|v_{n}\right|^{2}+\int_{\partial B_{m}}\left|\frac{\partial v_{n}}{\partial r}\right|\left|v_{n}\right|+\left|\int_{\partial D} \frac{\partial v_{n}}{\partial \eta} \bar{v}_{n}\right| \forall m>2,
$$

We claim that

$$
\lim _{n \rightarrow \infty} \int_{B_{m} \backslash D}\left|\nabla v_{n}\right|^{2} \leq C \quad \forall m>2 .
$$

Indeed, it suffices to prove that

$$
\lim _{n \rightarrow \infty} \int_{\partial \boldsymbol{B}_{m}}\left|\frac{\partial v_{n}}{\partial r}\right|\left|v_{n}\right| \leq C \quad \forall m>2 .
$$

We have

$$
\int_{\partial \boldsymbol{B}_{m}}\left|\frac{\partial v_{n}}{\partial r}\right|\left|v_{n}\right| \leq \int_{\partial \boldsymbol{B}_{m}}\left|\frac{\partial v_{0, n}}{\partial r}\right|\left|v_{n}\right|+\int_{\partial \boldsymbol{B}_{m}}\left|\frac{\partial v_{1, n}}{\partial r}\right|\left|v_{n}\right| .
$$

Since $\Delta v_{1, n}+\varepsilon_{n}^{2} v_{1, n}=0$, it follows from (2.13) and the standard theory of elliptic equations that

$$
\lim _{n \rightarrow \infty} \sup _{B_{2 m} \backslash B_{m}}\left(\left|v_{1, n}\right|+m\left|\nabla v_{1, n}\right|\right) \leq \frac{C}{\sqrt{m}} \quad \forall m>2 .
$$

On the other hand, from (2.5) and (2.12), one has

$$
\lim _{n \rightarrow \infty} \sup _{B_{2 m} \backslash \boldsymbol{B}_{m}} m\left|\frac{\partial v_{0, n}}{\partial r}\right|=0, \quad \lim _{n \rightarrow \infty} \sup _{B_{2 m} \backslash \boldsymbol{B}_{m}}\left|v_{0, n}\right| \leq C .
$$


Combining (2.17), (2.18), and (2.19) yields (2.16). Thus Claim (2.15) is proved.

From (2.14) and (2.15), we have

$$
\int_{\mathbb{R}^{2} \backslash D}|\nabla v|^{2}<+\infty
$$

Moreover, it follows from (2.8), (2.9), (2.12), and (2.18) that

$$
\begin{cases}\Delta v=0 & \text { in } \mathbb{R}^{2} \backslash \bar{D} \\ v=0 & \text { on } \partial D\end{cases}
$$

and

$$
\sup _{\mathbb{R}^{2} \backslash B_{1}}|v| \leq C
$$

Claim. We claim that

$$
v=0 \text {. }
$$

Proof of Claim: Fix $\phi \in C^{1}(\mathbb{R})$ such that $0 \leq \phi \leq 1, \phi=1$ if $|x| \leq 1$, and $\phi=0$ if $|x|>2$. Define

$$
\phi_{m}(x)=\phi(x / m) \quad \forall m>1 .
$$

Multiplying the first equation of (2.21) by $\bar{v} \phi_{m}$ and integrating the expression obtained on $\mathbb{R}^{2} \backslash D$, we have

$$
0=\int_{\mathbb{R}^{2} \backslash D} \nabla v \nabla\left(\bar{v} \phi_{m}\right)=\int_{\mathbb{R}^{2} \backslash D}|\nabla v|^{2} \phi_{m}+\int_{\mathbb{R}^{2} \backslash D} \bar{v} \nabla v \nabla \phi_{m} .
$$

However, since $\left|\nabla \phi_{m}\right| \leq C / m$ and $\operatorname{supp} \phi_{m} \subset B_{2 m} \backslash B_{m}$, it follows from (2.22) that

$$
\left|\int_{\mathbb{R}^{2} \backslash D} \bar{v} \nabla v \nabla \phi_{m}\right| \leq C\left(\int_{B_{2 m} \backslash B_{m}}|\nabla v|^{2}\right)^{\frac{1}{2}} .
$$

Hence combining (2.20) and (2.25) yields

$$
\lim _{m \rightarrow \infty} \int_{\mathbb{R}^{2} \backslash D} \bar{v} \nabla v \nabla \phi_{m}=0
$$

On the other hand, from the definition of $\phi_{m}$, we have

$$
\int_{\mathbb{R}^{2} \backslash D}|\nabla v|^{2} \leq \liminf _{m \rightarrow \infty} \int_{\mathbb{R}^{2} \backslash B}|\nabla v|^{2} \phi_{m} .
$$

Combining (2.24), (2.26), and (2.27), and letting $m$ go to infinity yields

$$
\int_{\mathbb{R}^{2} \backslash D}|\nabla v|^{2}=0
$$


Hence $v=0$ since $v=0$ on $\partial D$ and $\mathbb{R}^{2} \backslash D$ is connected. Claim (2.23) is proved.

Claim (2.23) contradicts the fact that $\|v\|_{L^{2}\left(B_{5} \backslash D\right)}=1$. Thus we obtain (2.7).

We next use (2.7) to prove (2.3). From (2.7) and decomposition (2.10), we have

$$
\left\|v_{\varepsilon}\right\|_{L^{2}\left(B_{r+1} \backslash D\right)} \leq C_{r}\|g\|_{H^{1 / 2}(\partial D)} .
$$

This implies, since $\Delta v_{\varepsilon}+\varepsilon^{2} v_{\varepsilon}=0$ in $B_{r+1} \backslash B_{r-1}$,

$$
\left\|v_{\varepsilon}\right\|_{H^{1 / 2}\left(\partial B_{r}\right)} \leq C_{r}\|g\|_{H^{1 / 2}(\partial D)} .
$$

Hence it follows from the standard theory of elliptic equations that

$$
\left\|v_{\varepsilon}\right\|_{H^{1}\left(B_{r} \backslash D\right)} \leq C_{r}\|g\|_{H^{1 / 2}(\partial D)} .
$$

It remains to prove (2.4). In fact, (2.4) is a consequence of (2.3) and decomposition (2.10).

The following lemma plays an important role in the proof of Theorem 2.1.

LEMMA 2.4 Let $d=2,3,0 \in D \subset B_{1}$ be a smooth, open, convex subset of $\mathbb{R}^{d}$, $0<\varepsilon<1$, a be a measurable matrix function, and $\sigma$ be a measurable complex function defined on $D_{1 / 2}$. Assume that a is uniformly elliptic, and $0 \leq \Im(\sigma)<$ $c_{1}<\infty$ and $0<c_{2}<\Re(\sigma)<c_{3}<\infty$ for some constants $c_{1}, c_{2}$, and $c_{3}$ on $D_{1 / 2}$. Let $g \in H^{-1 / 2}(\partial D)$ and $v_{\varepsilon} \in H_{\mathrm{loc}}^{1}\left(\mathbb{R}^{d}\right)$ be the unique solution of

$$
\begin{cases}\Delta v_{\varepsilon}+\varepsilon^{2} k^{2} v_{\varepsilon}=0 & \text { in } \mathbb{R}^{d} \backslash D, \\ \nabla \cdot\left(A \nabla v_{\varepsilon}\right)+k^{2} \Sigma v_{\varepsilon}=0 & \text { in } D, \\ \left.\frac{\partial v_{\varepsilon}}{\partial \eta}\right|_{\text {ext }}-\left.\frac{1}{\varepsilon^{d-2}} \frac{\partial v_{\varepsilon}}{\partial \eta}\right|_{\text {int }}=g & \text { on } \partial D, \\ v_{\varepsilon} \text { satisfies the outgoing condition. } & \end{cases}
$$

Here

$$
A=\left\{\begin{array}{ll}
I & \text { if } x \in \mathbb{R}^{d} \backslash D_{1 / 2}, \\
a & \text { if } x \in D_{1 / 2},
\end{array} \quad \Sigma= \begin{cases}1 & \text { if } x \in \mathbb{R}^{d} \backslash D, \\
{\left[1+\frac{i}{k}\right]} & \text { if } x \in D \backslash D_{1 / 2}, \\
\sigma & \text { if } x \in D_{1 / 2},\end{cases}\right.
$$

and $\eta$ is the unit normal vector on $\partial D$ directed into the exterior of $D$. Then

$$
\left\|v_{\varepsilon}\right\|_{H^{1}\left(B_{5} \backslash D\right)} \leq C \varepsilon^{d-2}\|g\|_{H^{-1 / 2}(\partial D)}
$$

for some positive constant $C$ independent of $\varepsilon, g, a, \sigma, c_{1}, c_{2}$, and $c_{3}$.

PROOF:

Step 1 . $d=3$. We first prove by contradiction that

$$
\left\|v_{\varepsilon}\right\|_{H^{1 / 2}(\partial D)} \leq C \varepsilon\|g\|_{H^{-1 / 2}(\partial D)} .
$$


Suppose that this is not true. Then there exists $\left(g_{n}\right) \subset H^{-1 / 2}(\partial D)$ and $\left(\varepsilon_{n}\right)$ such that $\varepsilon_{n}$ converges to 0 ,

$$
\left\|v_{n}\right\|_{H^{1 / 2}(\partial D)}=1 \quad \text { and } \quad \lim _{n \rightarrow \infty} \varepsilon_{n}\left\|g_{n}\right\|_{H^{-1 / 2}(\partial D)}=0,
$$

where $v_{n} \in H_{\text {loc }}^{1}\left(\mathbb{R}^{3}\right)$ is the unique solution of

$$
\begin{cases}\Delta v_{n}+\varepsilon_{n}^{2} k^{2} v_{n}=0 & \text { in } \mathbb{R}^{3} \backslash D, \\ \nabla \cdot\left(A_{n} \nabla v_{n}\right)+k^{2} \Sigma_{n} v_{n}=0 & \text { in } D, \\ \left.\frac{\partial v_{n}}{\partial \eta}\right|_{\text {ext }}-\left.\frac{1}{\varepsilon_{n}} \frac{\partial v_{n}}{\partial \eta}\right|_{\text {int }}=g_{n} & \text { on } \partial D, \\ v_{n} \text { satisfies the outgoing condition. } & \end{cases}
$$

Here $A_{n}$ and $\Sigma_{n}$ are defined similarly as $A$ and $\Sigma$. However, $a$ and $\sigma$ are replaced by $a_{n}$ and $\sigma_{n}$ for some $\left(a_{n}\right)$ and $\left(\sigma_{n}\right)$ satisfying the standard conditions.

Since $\left\|v_{n}\right\|_{H^{1 / 2}(\partial D)}=1$, by Lemma 2.2 , we have

$$
\left\|v_{n}\right\|_{H^{1}\left(B_{5} \backslash D\right)} \leq C \text {. }
$$

This implies

$$
\lim _{n \rightarrow \infty}\left\|\left.\frac{\partial v_{n}}{\partial \eta}\right|_{\text {int }}\right\|_{H^{-1 / 2}(\partial D)}=0 .
$$

Multiplying system (2.30) by $\bar{v}_{n}$ (the conjugate of $v_{n}$ ) and integrating the expression obtained over $B_{4}$, one has

$$
\begin{array}{r}
\int_{B_{4} \backslash D}\left|\nabla v_{n}\right|^{2}-\varepsilon_{n}^{2} k^{2} \int_{B_{4} \backslash D}\left|v_{n}\right|^{2}+\frac{1}{\varepsilon_{n}} \int_{D}\left\langle A_{n} \nabla v_{n}, \nabla v_{n}\right\rangle-\frac{1}{\varepsilon_{n}} \int_{D} k^{2} \Sigma_{n}\left|v_{n}\right|^{2}= \\
\int_{\partial B_{4}} \frac{\partial v_{n}}{\partial r} \bar{v}_{n}-\int_{\partial D} g_{n} \bar{v}_{n} .
\end{array}
$$

Considering the imaginary part, from (2.31), one has

$$
\lim _{n \rightarrow \infty} \int_{D \backslash D_{1 / 2}}\left|v_{n}\right|^{2}=0 .
$$

Multiplying system (2.30) with $\bar{v}_{n}$, integrating the expression obtained over $D \backslash$ $D_{\delta}$ with respect to $x$ and then integrating the expression obtained with respect to $\delta$ on $\left[\frac{3}{5}, \frac{2}{3}\right]$, we have

$$
\begin{aligned}
\int_{3 / 5}^{2 / 3} \int_{D \backslash D_{\delta}}\left|\nabla v_{n}\right|^{2}-\int_{3 / 5}^{2 / 3} \int_{D \backslash D_{\delta}} k^{2} \Sigma_{n}\left|v_{n}\right|^{2}= \\
\left.\left.\frac{1}{15} \int_{\partial D} \frac{\partial v_{n}}{\partial \eta}\right|_{\text {int }} \bar{v}_{n}\right|_{\text {int }}-\int_{3 / 5}^{2 / 3} \int_{\partial D_{\delta}} \frac{\partial v_{n}}{\partial \eta} \bar{v}_{n} .
\end{aligned}
$$


Hence, since $\Delta v_{n}+k^{2} \Sigma v_{n}=0$ in $D \backslash D_{1 / 2}$, it follows from (2.32) and (2.33) that

$$
\lim _{n \rightarrow \infty} \int_{D \backslash D_{3 / 4}}\left|\nabla v_{n}\right|^{2}=0
$$

this implies

$$
\lim _{n \rightarrow \infty}\left\|v_{n}\right\|_{H^{1 / 2}(\partial D)}=0 .
$$

We have a contradiction. Thus (2.29) holds. The conclusion now follows from Lemma 2.2.

Step 2. $d=2$. We first prove by contradiction that

$$
\left\|v_{\varepsilon}\right\|_{L^{2}\left(B_{5} \backslash D\right)} \leq C\|g\|_{H^{-1 / 2}(\partial D)}
$$

Suppose that this is not true. Then there exists $\left(g_{n}\right) \subset H^{-1 / 2}(\partial D)$ and $\left(\varepsilon_{n}\right)$ such that $\varepsilon_{n}$ converges to 0 ,

$$
\left\|v_{n}\right\|_{L^{2}\left(B_{5} \backslash D\right)}=1 \quad \text { and } \quad \lim _{n \rightarrow \infty}\left\|g_{n}\right\|_{H^{-1 / 2}(\partial D)}=0,
$$

where $v_{n} \in H_{\text {loc }}^{1}\left(\mathbb{R}^{2}\right)$ is the unique solution of

$$
\begin{cases}\Delta v_{n}+\varepsilon_{n}^{2} k^{2} v_{n}=0 & \text { in } \mathbb{R}^{2} \backslash D, \\ \nabla \cdot\left(A_{n} \nabla v_{n}\right)+k^{2} \Sigma_{n} v_{n}=0 & \text { in } D, \\ \left.\frac{\partial v_{n}}{\partial \eta}\right|_{\text {ext }}-\left.\frac{\partial v_{n}}{\partial \eta}\right|_{\text {int }}=g_{n} & \text { on } \partial D, \\ v_{n} \text { satisfies the outgoing condition. } & \end{cases}
$$

Here $A_{n}$ and $\Sigma_{n}$ are defined similarly to $A$ and $\Sigma$. However, $a$ and $\sigma$ are replaced by $a_{n}$ and $\sigma_{n}$ for some $\left(a_{n}\right)$ and $\left(\sigma_{n}\right)$ satisfying the standard conditions.

Multiplying system (2.36) by $\bar{v}_{n}$ and integrating the expression obtained over $B_{R}(R$ large), one has

$$
\begin{array}{r}
\int_{B_{R} \backslash D}\left|\nabla v_{n}\right|^{2}-\varepsilon_{n}^{2} k^{2} \int_{B_{R} \backslash D}\left|v_{n}\right|^{2}+\int_{D}\left\langle A_{n} \nabla v_{n}, \nabla v_{n}\right\rangle-\int_{D} k^{2} \Sigma_{n}\left|v_{n}\right|^{2}= \\
\int_{\partial B_{R}} \frac{\partial v_{n}}{\partial r} \bar{v}_{n}-\int_{\partial D} g_{n} \bar{v}_{n} .
\end{array}
$$

Letting $R$ go to infinity, using the outgoing condition, and considering the imaginary part, one obtains

$$
\int_{D \backslash D_{1 / 2}}\left|v_{n}\right|^{2} \leq C\left\|g_{n}\right\|_{H^{-1 / 2}(\partial D)}\left\|v_{n}\right\|_{H^{1 / 2}(\partial D)} .
$$


Multiplying system (2.36) by $\bar{v}_{n}$ and integrating the expression obtained over $B_{4} \backslash$ $D_{2 / 3}$, one has

$$
\begin{array}{r}
\int_{B_{4} \backslash D}\left|\nabla v_{n}\right|^{2}-\varepsilon_{n}^{2} k^{2} \int_{B_{4} \backslash D}\left|v_{n}\right|^{2}+\int_{D \backslash D_{2 / 3}}\left\langle A_{n} \nabla v_{n}, \nabla v_{n}\right\rangle-\int_{D \backslash D_{2 / 3}} k^{2} \Sigma_{n}\left|v_{n}\right|^{2}= \\
\int_{\partial B_{4}} \frac{\partial v_{n}}{\partial r} \bar{v}_{n}-\int_{\partial D} g_{n} \bar{v}_{n}-\int_{\partial D_{2 / 3}} \frac{\partial v_{n}}{\partial \eta} \bar{v}_{n} .
\end{array}
$$

Hence, by Lemma 2.2 it follows from (2.35) and (2.37) that

$$
\left\|\nabla v_{n}\right\|_{L^{2}\left(B_{4} \backslash D_{2 / 3}\right)} \leq C .
$$

Combining (2.35), (2.37), and (2.38) and applying Lemma 2.2 yields

$$
\left\|v_{n}\right\|_{H^{1}\left(B_{5} \backslash D_{2 / 3}\right)} \leq C
$$

and

$$
\lim _{n \rightarrow \infty} \int_{D \backslash D_{2 / 3}}\left|v_{n}\right|^{2}=0
$$

Applying Lemma 2.2, without loss of generality, one may assume that $v_{n} \rightarrow v$ weakly in $H_{\text {loc }}^{1}\left(\mathbb{R}^{2} \backslash D_{2 / 3}\right)$ and $v_{n} \rightarrow v$ in $L^{2}\left(B_{5} \backslash D\right)$. Here $v \in H_{\text {loc }}^{1}\left(\mathbb{R}^{2} \backslash D_{2 / 3}\right)$ satisfies

$$
\begin{cases}\Delta v+\left(k^{2}+i k\right) \mathbb{1}_{D \backslash D_{2 / 3}} v=0 & \text { in } \mathbb{R}^{2} \backslash \overline{D_{2 / 3}}, \\ v=0 & \text { in } D \backslash D_{2 / 3} .\end{cases}
$$

Here $\mathbb{1}_{A}$ denotes the characteristic function of any set $A$ of $\mathbb{R}^{2}$. This implies $v=0$. We have a contradiction since $\left\|v_{n}\right\|_{L^{2}\left(B_{5} \backslash D\right)}=1$. Thus (2.35) is proved. The conclusion now follows by applying the argument used to obtain (2.39).

We are ready to give the following proof:

PROOF OF THEOREM 2.1: Let $u_{1, \varepsilon} \in H_{\mathrm{loc}}^{1}\left(\mathbb{R}^{d}\right)$ be the unique solution of

$$
\begin{cases}\Delta u_{1, \varepsilon}+k^{2} u_{1, \varepsilon}=f & \text { in } \mathbb{R}^{d} \backslash D_{\varepsilon}, \\ u_{1, \varepsilon}=0 & \text { in } D_{\varepsilon}, \\ u_{1, \varepsilon} \text { satisfies the outgoing condition. } & \end{cases}
$$

Define

$$
w_{1, \varepsilon}=u_{1, \varepsilon}-u \quad \text { and } \quad w_{2, \varepsilon}=u_{\varepsilon}-u_{1, \varepsilon} .
$$

We claim that

$$
\begin{cases}\left\|w_{1, \varepsilon}\right\|_{H^{1}\left(B_{3} \backslash B_{2}\right)} \leq \frac{C}{\mid \ln \varepsilon}\|f\|_{L^{2}} & \text { if } d=2, \\ \left\|w_{1, \varepsilon}\right\|_{H^{1}\left(B_{3} \backslash B_{2}\right)} \leq C \varepsilon\|f\|_{L^{2}} & \text { if } d=3,\end{cases}
$$


and

$$
\begin{cases}\left\|w_{2, \varepsilon}\right\|_{H^{1}\left(B_{3} \backslash B_{2}\right)} \leq \frac{C}{\mid \ln \varepsilon}\|f\|_{L^{2}} & \text { if } d=2, \\ \left\|w_{2, \varepsilon}\right\|_{H^{1}\left(B_{3} \backslash B_{2}\right)} \leq C \varepsilon^{2}\|f\|_{L^{2}} & \text { if } d=3 .\end{cases}
$$

PROOF OF (2.41): From the definition of $w_{1, \varepsilon}$, it follows that $w_{1, \varepsilon} \in H_{\mathrm{loc}}^{1}\left(\mathbb{R}^{d}\right)$ and $w_{1, \varepsilon}$ satisfies

$$
\begin{cases}\Delta w_{1, \varepsilon}+k^{2} w_{1, \varepsilon}=0 & \text { in } \mathbb{R}^{d} \backslash D_{\varepsilon} \\ w_{1, \varepsilon}=-u & \text { in } \partial D_{\varepsilon} \\ w_{1, \varepsilon} \text { satisfies the outgoing condition. } & \end{cases}
$$

Define $W_{1, \varepsilon}(x)=w_{1, \varepsilon}(\varepsilon x)$. Then $W_{1, \varepsilon} \in H_{\text {loc }}^{1}\left(\mathbb{R}^{d}\right)$ and $W_{1, \varepsilon}$ satisfies

$$
\begin{cases}\Delta W_{1, \varepsilon}+\varepsilon^{2} k^{2} W_{1, \varepsilon}=0 & \text { in } \mathbb{R}^{d} \backslash D, \\ W_{1, \varepsilon}=-u(\varepsilon \cdot) & \text { in } \partial D, \\ W_{1, \varepsilon} \text { satisfies the outgoing condition. } & \end{cases}
$$

Since $\|u(\varepsilon \cdot)\|_{H^{1 / 2}(\partial D)} \leq\|f\|_{L^{2}},(2.41)$ follows from Lemma 2.2.

Proof OF (2.42): It is clear that $w_{2, \varepsilon} \in H_{\mathrm{loc}}^{1}\left(\mathbb{R}^{d}\right)$ is the unique solution of

$$
\begin{cases}\Delta w_{2, \varepsilon}+k^{2} w_{2, \varepsilon}=0 & \text { in } \mathbb{R}^{d} \backslash D_{\varepsilon}, \\ \nabla \cdot\left(A_{\varepsilon} \nabla w_{2, \varepsilon}\right)+k^{2} \Sigma_{\varepsilon} w_{2, \varepsilon}=0 & \text { in } D_{\varepsilon}, \\ \left.\frac{\partial w_{2, \varepsilon}}{\partial \eta}\right|_{\text {ext }}-\left.\frac{1}{\varepsilon^{d-2}} \frac{\partial w_{2, \varepsilon}}{\partial \eta}\right|_{\text {int }}=-\frac{\partial u_{1, \varepsilon}}{\partial \eta} & \text { on } \partial D_{\varepsilon} \\ w_{2, \varepsilon} \text { satisfies the outgoing condition. } & \end{cases}
$$

Define $W_{2, \varepsilon}(x)=w_{2, \varepsilon}(\varepsilon x)$. Then $W_{2, \varepsilon} \in H_{\mathrm{loc}}^{1}\left(\mathbb{R}^{d}\right)$ and $W_{2, \varepsilon}$ is the unique solution of

$$
\begin{cases}\Delta W_{2, \varepsilon}+\varepsilon^{2} k^{2} W_{2, \varepsilon}=0 & \text { in } \mathbb{R}^{d} \backslash D, \\ \nabla \cdot\left(A \nabla W_{2, \varepsilon}\right)+k^{2} \Sigma W_{2, \varepsilon}=0 & \text { in } D, \\ \left.\frac{\partial W_{2, \varepsilon}}{\partial \eta}\right|_{\text {ext }}-\left.\frac{1}{\varepsilon^{d-2}} \frac{\partial W_{2, \varepsilon}}{\partial \eta}\right|_{\text {int }}=-\varepsilon \frac{\partial u_{1, \varepsilon}}{\partial \eta}(\varepsilon x) & \text { on } \partial D \\ W_{2, \varepsilon} \text { satisfies the outgoing condition. } & \end{cases}
$$

Here

$$
A=\left\{\begin{array}{ll}
I & \text { if } x \in \mathbb{R}^{d} \backslash D_{1 / 2}, \\
a(x) & \text { if } x \in D_{1 / 2},
\end{array} \quad \sigma= \begin{cases}1 & \text { if } x \in \mathbb{R}^{d} \backslash D, \\
{\left[1+\frac{i}{k}\right]} & \text { if } x \in D \backslash D_{1 / 2}, \\
\sigma(x) & \text { if } x \in D_{1 / 2} .\end{cases}\right.
$$

Applying Lemma 2.4, since $u_{1, \varepsilon}=w_{1, \varepsilon}+u$ and $W_{1, \varepsilon}=w_{1, \varepsilon}(\varepsilon \cdot)$, by Lemma 2.2, we have

$$
\left\|W_{2, \varepsilon}\right\|_{H^{1}\left(B_{5} \backslash D\right)} \leq C \varepsilon^{d-2}\|f\|_{L^{2}} .
$$

Thus since $w_{2, \varepsilon}(x)=W_{2, \varepsilon}(x / \varepsilon)$, claim (2.42) follows from Lemma 2.2. 
Thus our claims (2.41) and (2.42) are proved. Therefore, since $u_{\varepsilon}-u=w_{1, \varepsilon}+$ $w_{2, \varepsilon}$, the conclusion follows.

Remark 2.5. The idea of splitting $u_{\varepsilon}-u$ into $w_{1, \varepsilon}$ and $w_{2, \varepsilon}$ originally appeared in the work of Nguyen and Vogelius [14], where the authors establish the uniform expansions of the Neumann-to-Dirichlet map in the context of electric impedance tomography under the perturbation of small inclusions.

\subsection{Necessity of a Lossy Layer; Proof of Theorem 1.7}

We recall that near-cloaking achieved without a lossy layer was discussed in some cases (see, e.g., [16]) when the material inside the cloaked region is fixed. In this section we will show that, by a direct calculation, this statement is false if no requirement on the properties of the material inside the cloaked region is imposed. In fact, we will show that we can choose the material inside the cloaked region such that the scattered wave is large no matter how small $\varepsilon$ is.

Define

$$
A= \begin{cases}I & \text { in } \mathbb{R}^{d} \backslash B_{\varepsilon} \\ c I & \text { in } B_{\varepsilon}\end{cases}
$$

and

$$
\Sigma= \begin{cases}1 & \text { in } \mathbb{R}^{d} \backslash B_{\varepsilon} \\ c q & \text { in } B_{\varepsilon} .\end{cases}
$$

Let $u_{s} \in H_{\text {loc }}^{1}\left(\mathbb{R}^{d}\right)$ be such that $u_{s}$ satisfies the radiation condition and $u:=$ $u_{s}+e^{i k x_{d}}$ is the solution of

$$
\operatorname{div}(A \nabla u)+k^{2} \Sigma u=0 \quad \text { in } \mathbb{R}^{d} .
$$

The necessity of having a lossy layer stated in Theorem 1.7 is a consequence of Proposition 1.1 and the following result:

THEOREM 2.6 Let $d=2$, 3. Then for any $0<\varepsilon<2, r>4$, and $\lambda>0$, there exist $q>0$ and $c>0$ such that

$$
\left\|u_{S}\right\|_{L^{2}\left(B_{r} \backslash B_{2}\right)} \geq \lambda .
$$

PROOF: We only prove the proposition for the case $\varepsilon=1$ and $k=1$. The general case follows by the same argument.

Case 1. $d=3$. We have (see, e.g., [2, (2.45), p. 32]),

$$
u_{i}:=e^{i x_{3}}=\sum_{n=0}^{\infty} c_{n} j_{n}(|x|) Y_{n}^{0}(\hat{x}),
$$


where $\left|c_{n}\right|=\sqrt{4 \pi(2 n+1)}, Y_{n}^{0}(\theta, \varphi)=\sqrt{(2 n+1) / 4 \pi} P_{n}(\cos \theta)\left(P_{n}\right.$ is the Legendre polynomial of order $n)$,

$$
\begin{array}{ll}
u_{s}=\sum_{n=0}^{\infty} \alpha_{n} h_{n}(|x|) Y_{n}^{0}(\hat{x}) & \text { for }|x|>1, \\
u_{t}=\sum_{n=0}^{\infty} \beta_{n} j_{n}(\sqrt{q}|x|) Y_{n}^{0}(\hat{x}) & \text { for }|x|<1,
\end{array}
$$

where $u_{t}:=u_{s}+u_{i}$ in $B_{1}$. Here $h_{n}$ is the spherical Hankel function of the first kind of order $n$ and $j_{n}=\Re\left(h_{n}\right)$.

Using the transmission conditions, namely,

$$
\begin{cases}u_{s}+u_{i}=u_{t} & \text { on } \partial B_{1}, \\ \frac{\partial u_{s}}{\partial r}+\frac{\partial u_{i}}{\partial r}=c \frac{\partial u_{t}}{\partial r} & \text { on } \partial B_{1}\end{cases}
$$

we have

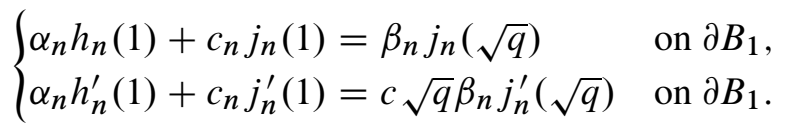

Thus it follows that

$$
\alpha_{n}=B_{n} c_{n}
$$

where

$$
B_{n}=-\frac{j_{n}^{\prime}(1) j_{n}(\sqrt{q})-c \sqrt{q} j_{n}(1) j_{n}^{\prime}(\sqrt{q})}{h_{n}^{\prime}(1) j_{n}(\sqrt{q})-c \sqrt{q} h_{n}(1) j_{n}^{\prime}(\sqrt{q})} .
$$

Let $n$ be large. Then by [2, (2.38), p. 29],

$$
y_{n}(t)=-\frac{1 \cdots(2 n-1)}{t^{n+1}}(1+O(1 / n))
$$

and

$$
y_{n}^{\prime}(t)=\frac{n+1}{t} \cdot \frac{1 \cdots(2 n-1)}{t^{n+1}}(1+O(1 / n)) .
$$

Here $y_{n}=\Im\left(h_{n}\right)$, i.e., $h_{n}=j_{n}+i y_{n}$. Thus

$$
\frac{y_{n}^{\prime}(1)}{y_{n}(1)}=-(n+1)(1+O(1 / n)) \text {. }
$$

Fix such an $n$. Take $\sqrt{q} \gg n$. Then, by [2, (2.41), p. 30], we have

$$
j_{n}(\sqrt{q})=\frac{1}{\sqrt{q}} \cos \left(\sqrt{q}-\frac{n \pi}{2}-\frac{\pi}{2}\right)(1+O(1 / \sqrt{q}))
$$

and

$$
j_{n}^{\prime}(\sqrt{q})=-\frac{1}{\sqrt{q}} \sin \left(\sqrt{q}-\frac{n \pi}{2}-\frac{\pi}{2}\right)(1+O(1 / \sqrt{q})) .
$$

Hence it follows that

$$
c \sqrt{q} \frac{j_{n}^{\prime}(\sqrt{q})}{j_{n}(\sqrt{q})}=-c \sqrt{q} \frac{\sin \left(\sqrt{q}-\frac{n \pi}{2}-\frac{\pi}{2}\right)}{\cos \left(\sqrt{q}-\frac{n \pi}{2}-\frac{\pi}{2}\right)}(1+O(1 / \sqrt{q})) .
$$


Thus there exist $q$ and $c$ such that

$$
\frac{y_{n}^{\prime}(1)}{j_{n}(1)}=c \sqrt{q} \frac{y_{n}^{\prime}(\sqrt{q})}{j_{n}(\sqrt{q})}
$$

Take such $q$ and $c$. Then

$$
B_{n}=-1
$$

which implies

$$
\alpha_{n}=-c_{n}
$$

On the other hand,

$$
h_{n}(t)=\frac{1 \cdots(2 n-1)}{i t^{n+1}}(1+O(1 / n)) \quad \text { as } n \rightarrow \infty .
$$

Thus the conclusion follows.

Case 2. $d=2$. The proof in this case is similar to the case $d=3$. We have (see, e.g., [2, (3.66), p. 67]),

$$
\begin{gathered}
u_{i}:=e^{i x_{2}}=\sum_{n=-\infty}^{\infty} c_{n} J_{n}(|x|) e^{i n \theta}, \\
u_{s}=\sum_{n=-\infty}^{\infty} \alpha_{n} H_{n}(|x|) e^{i n \theta} \quad \text { for }|x|>1,
\end{gathered}
$$

and

$$
u_{t}=\sum_{n=-\infty}^{\infty} \beta_{n} J_{n}(\sqrt{q}|x|) e^{i n \theta} \quad \text { for }|x|<1,
$$

where $\left|c_{n}\right|=1, u_{t}:=u_{s}+u_{i}$ in $B_{1}$. Here $H_{n}$ is the Hankel function of the first kind of order $n$ and $J_{n}=\Re\left(H_{n}\right)$.

Using the transmission conditions, as in the case $d=3$, we also have

$$
\alpha_{n}=B_{n} c_{n},
$$

where

$$
B_{n}=-\frac{J_{n}^{\prime}(1) J_{n}(\sqrt{q})-c \sqrt{q} J_{n}(1) J_{n}^{\prime}(\sqrt{q})}{H_{n}^{\prime}(1) J_{n}(\sqrt{q})-c \sqrt{q} H_{n}(1) J_{n}^{\prime}(\sqrt{q})} .
$$

Let $n$ be large. Then by [2, (3.58), p. 66],

$$
Y_{n}(t)=-\frac{2^{n}(n-1) !}{\pi t^{n}}(1+O(1 / n))
$$

and

$$
Y_{n}^{\prime}(t)=\frac{2^{n} n !}{\pi t^{n+1}}(1+O(1 / n)) .
$$

Here $Y_{n}=\Im\left(H_{n}\right)$, i.e., $H_{n}=J_{n}+i Y_{n}$. Thus

$$
\frac{Y_{n}^{\prime}(1)}{Y_{n}(1)}=-n(1+O(1 / n)) .
$$


Fix such an $n$. Take $\sqrt{q} \gg n$. Then, from [2, (3.59), p. 66],

$$
J_{n}(\sqrt{q})=\sqrt{\frac{2}{\pi \sqrt{q}}} \cos \left(\sqrt{q}-\frac{n \pi}{2}-\frac{\pi}{4}\right)(1+O(1 / \sqrt{q}))
$$

and

$$
J_{n}^{\prime}(\sqrt{q})=\sqrt{\frac{2}{\pi \sqrt{q}}} \cos \left(\sqrt{q}-\frac{n \pi}{2}+\frac{\pi}{4}\right)(1+O(1 / \sqrt{q})) .
$$

Hence it follows that

$$
c \sqrt{q} \frac{J_{n}^{\prime}(\sqrt{q})}{J_{n}(\sqrt{q})}=c \sqrt{q} \frac{\cos \left(\sqrt{q}-\frac{n \pi}{2}-\frac{\pi}{4}\right)}{\cos \left(\sqrt{q}-\frac{n \pi}{2}+\frac{\pi}{4}\right)}(1+O(1 / \sqrt{q})) .
$$

Thus there exist $q$ and $c$ such that

$$
\frac{Y_{n}^{\prime}(1)}{J_{n}(1)}=c \sqrt{q} \frac{Y_{n}^{\prime}(\sqrt{q})}{J_{n}(\sqrt{q})}
$$

Take such $q$ and $c$. Then

$$
B_{n}=-1 \text {, }
$$

which implies

$$
\alpha_{n}=-c_{n}
$$

On the other hand,

$$
H_{n}(t)=\frac{2^{n}(n-1) !}{\pi i t^{n}}(1+O(1 / n)) \quad \text { as } n \rightarrow \infty .
$$

Thus the conclusion follows.

Acknowledgment. The author would like to thank R. V. Kohn for suggesting the problem, pointing out relevant papers, and stimulating discussions. The author also wishes to thank him for reading the manuscript and commenting on it. This greatly improved the presentation of the paper.

\section{Bibliography}

[1] Colton, D.; Kress, R. Integral equation methods in scattering theory. Pure and Applied Mathematics (New York). Wiley, New York, 1983.

[2] Colton, D.; Kress, R. Inverse acoustic and electromagnetic scattering theory. 2nd ed. Applied Mathematical Sciences, 93. Springer, Berlin, 1998.

[3] Greenleaf, A.; Kurylev, Y.; Lassas, M.; Uhlmann, G. Full-wave invisibility of active devices at all frequencies. Comm. Math. Phys. 275 (2007), no. 3, 749-789.

[4] Greenleaf, A.; Kurylev, Y.; Lassas, M.; Uhlmann, G. Invisibility and inverse problems. Bull. Amer. Math. Soc. (N.S.) 46 (2009), no. 1, 55-97.

[5] Greenleaf, A.; Lassas, M.; Uhlmann, G. On nonuniqueness for Calderón's inverse problem. Math. Res. Lett. 10 (2003), no. 5-6, 685-693.

[6] Hetmaniuk, U.; Liu, H. Y.; Uhlmann, G. On three dimensional active acoustic cloaking devices and their simulation. Preprint. Available at: http://www.math.washington.edu/ $\sim$ hyliu/Preprints/3D_Acoustic_Cloaking.pdf 
[7] Kohn, R. V.; Onofrei, D.; Vogelius, M. S.; Weinstein, M. I. Cloaking via change of variables for the Helmholtz equation. Comm. Pure Appl. Math. 63 (2010), no. 8, 973-1016.

[8] Kohn, R. V.; Shen, H.; Vogelius, M. S.; Weinstein, M. I. Cloaking via change of variables in electric impedance tomography. Inverse Problems 24 (2008), no. 1, 015016, 21 pp.

[9] Kress, R. On the low wave number asymptotics for the two-dimensional exterior Dirichlet problem for the reduced wave equation. Math. Methods Appl. Sci. 9 (1987), no. 3, 335-341.

[10] Leis, R. Initial-boundary value problems in mathematical physics. Teubner, Stuttgart; Wiley, Chichester, 1986.

[11] Leonhardt, U. Optical conformal mapping. Science 312 (2006), no. 5781, 1777-1780.

[12] Liu, H. Virtual reshaping and invisibility in obstacle scattering. Inverse Problems 25 (2009), no. $4,045006,16 \mathrm{pp}$.

[13] Nédélec, J. C. Acoustic and electromagnetic equations. Integral representations for harmonic problems. Applied Mathematical Sciences, 144. Springer, Berlin, 2001.

[14] Nguyen, H-M.; Vogelius, M. S. A representation formula for the voltage perturbations caused by diametrically small conductivity inhomogeneities. Proof of uniform validity. Ann. Inst. H. Poincaré Anal. Non Linéaire 26 (2009), 2283-2315. Available at: http: //cims.nyu. edu/ hoaiminh/publication.php

[15] Pendry, J. B.; Schurig, D.; Smith, D. R. Controlling electromagnetic fields. Science 312 (2006), no. $5781,1780-1782$.

[16] Ruan, Z.; Yan, M.; Neff, C. M.; Qiu, M. Ideal cylindrical cloak: perfect but sensitive to tiny perturbations. Phys. Rev. Lett. 99 (2007), 113903, 4 pp.

[17] Watson, G. N. A treatise on the theory of Bessel functions. Cambridge University Press, Cambridge, England; Macmillan, New York, 1944.

[18] Weder, R. The boundary conditions for point transformed electromagnetic invisibility cloaks. J. Phys. A 41 (2008), no. 41, 415401, 17 pp.

[19] Weder, R. A rigorous analysis of high-order electromagnetic invisibility cloaks. J. Phys. A 41 (2008), no. 6, 065207, 21 pp.

[20] Werner, P. Low frequency asymptotics for the reduced wave equation in two-dimensional exterior spaces. Math. Methods Appl. Sci. 8 (1986), no. 1, 134-156.

[21] Wilcox, C. H. Scattering theory for the d'Alembert equation in exterior domains. Lecture Notes in Mathematics, 442. Springer, Berlin-New York, 1975.

Hoai-Minh NguYen

Courant Institute

251 Mercer Street

New York, NY 10012

E-mail: hoaiminhecims.nyu.edu

Received October 2009. 